Check for updates

Cite this: RSC Adv., 2017, 7, 24242

\title{
A one-step process employing various amphiphiles for an electrically insulating silica coating on graphite $\uparrow$
}

\author{
Yeongseon Kim, Yingjie Qian, Minjae Kim, Jaechul Ju, Sung-Hyeon Baeck \\ and Sang Eun Shim (D) *
}

Ceramic coatings endow carbon materials with electrically insulating properties. For graphite, it is unclear whether ceramic coatings applied via a one-step process under mild conditions can lead to superior coverage. This paper reports that a modified Stöber method with an appropriate choice of amphiphiles could yield an electrically insulating coating layer on graphite $\left(d_{50} 3-300 \mu \mathrm{m}, d_{90} 6-550 \mu \mathrm{m}\right)$ within $24 \mathrm{~h}$ with high reproducibility. A silica coating mechanism involving functional groups on the edge of the graphite, Oswald ripening agents, and a bridgemer was investigated. The mechanism was based on the silica coating morphology, which depends on the amphiphile, and the correlation between coverage and surface resistivity was assessed. Amphiphile-assisted silica@graphite with a surface resistivity of $10^{12}$ $\mathrm{ohm} \mathrm{sq}{ }^{-1}$ was produced. The thermal conductivity of the silica-coated graphite (amphiphile-assisted silica@graphite)/TPEE composite reached values over 75\% higher than that of the raw graphite/TPEE composite with electrically insulating properties.

Received 14th March 2017

Accepted 28th April 2017

DOI: $10.1039 / c 7 r a 03049 e$

rsc.li/rsc-advances graphite is under $10^{2} \mathrm{ohm} \mathrm{sq}^{-1}$. This limits their applicability as an additive to the coating solution for printed circuit boards (PCBs), an adhesive for the assembly of electronic chips, pigments for thermal paints, composites fillers for cable coverings, and packaging materials for electronics. ${ }^{6}$

Surface modification methods for carbon materials have helped them achieve superior potential and reliable properties (i.e., mechanical, electrical, and thermal properties) in carbon fillers. Methods for improving the physical and chemical affinity between fillers and polymer matrices affect the distribution/dispersion state of the filler networks. These improvement methods include surface functionalization (i.e., grafting functional groups on/from the surface of the carbon), dispersant treatment, coupling agent treatment, and the decoration method. The percolation threshold for the filler loading in a polymer matrix is shifted to a lower level..$^{7-15}$ On the other hand, severe surface modification methods such as acid treatments and physical impacts degrade the original properties of the carbon, because these methods damage the conjugated $\mathrm{sp}^{2}$ structures of the planar moieties.

Coating a amphiphile-covered carbon substrate with a highcoverage ceramic layer is a suitable method for overcoming the abovementioned drawbacks of the carbon material, while avoiding the formation of defects via harsh modification methods. Such a method offers transforms to the electrical properties of carbon fillers, and they can possess thermally conductive and electrically insulating properties via this strategy. ${ }^{16-23}$ In previous studies, a dense silica layer was successfully coated onto graphite via a polyvinylpyrrolidone 
(PVP)-assisted sol-gel reaction under basic conditions. PVP played a crucial role in overcoming poor chemical affinity between the hydrophilic silica and hydrophobic planar moieties of the graphite surface. The PVP-assisted silica@graphite was categorized as an electrical insulator with a high surface resistivity of up to $10^{12} \mathrm{ohm} \mathrm{sq}{ }^{-1}$; nevertheless, the PVP-assisted silica@graphite has a problem in terms of productivity. The process consists of two steps: the physical adsorption of PVP on graphite in $\mathrm{H}_{2} \mathrm{O}$, and the formation of a silica layer on PVP@graphite in ethanol. ${ }^{18,19} \mathrm{~A}$ work-up process that included filtration and drying was involved in both steps. As a result, the two-step process required over $40 \mathrm{~h}$ for processing.

There exist a few results on the use of alumina and silica coated graphite for thermally conductive composites. However, the synthetic conditions, synthetic mechanism or genuine properties of the as-prepared materials, which strongly influence the thermal and electrical transporting characteristics, have not been scrutinized well to date. ${ }^{22,23}$

Herein, one-step processes that fully covers graphite $\left(d_{50} 3-\right.$ $300 \mu \mathrm{m}, d_{90} 6-550 \mu \mathrm{m}$ ) with a silica layer within $12-25 \mathrm{~h}$, are suggested. Fig. 1 presents the basic concept of the amphiphileassisted silica@graphite (Grasil), with a correlation between each silica coating procedure for silica coating and the resulting properties (i.e., thermal conductivity and surface resistivity). Although covering raw graphite with an amphiphile causes a minuscule decrease in its thermal conductivity, the amphiphile provides a hydrophobic basal plane with functional groups. The hydroxyl groups of the enol tautomer of $\mathrm{PVP}^{19}$ (Triton X-100, SDS, ${ }^{25}$ lignin, ${ }^{26}$ and $\mathrm{PEG}^{27}$ ) are appropriate for inducing a dehydration reaction in the silanol group of a silica precursor. The growth of polysilicate species under basic conditions results in branched structures, which have a broader distribution. In contrast, the growth of polysilicate species under acidic conditions is predisposed to form a linear structure. ${ }^{28}$ The abundance of growing points (i.e., secondary, tertiary) of nuclei has the advantage of the covering work; it can facilitate the growth of silica species in the immediate vicinity of existing nuclei. A thin silica layer coated on the outside is considered a thermal and electrical barrier (the thermal conductivity of silica is $\left.1.5 \mathrm{~W} \mathrm{mK}^{-1}\right),{ }^{29}$ which endows raw graphite with electrically insulating properties. Fig. 2 illustrates how to approach the modified one-step process, and reduce the

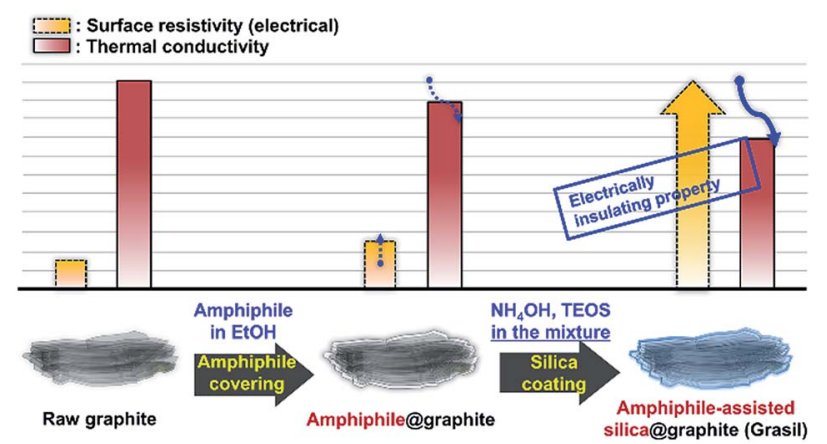

Fig. 1 Basic concept for the amphiphile-assisted silica@graphite (Grasil) via the modified one-step process. processing time to less than $50 \%$ of existing two-step process. The media were unified as ethanol under basic conditions. There are several considerations for encouraging appropriate amphiphile absorption on graphite for silica growth. Hightemperature treatment at $500{ }^{\circ} \mathrm{C}$ reduces drying time to less than $50 \%$ of its original value, and reinforces the mechanical properties of the silica layer (thermal sintering). PEG is applied to the modified one-step process, and helps reduce the coatingprocess time under one-fourth (ver. 2).

This paper aimed to provide thermally conductive and electrically insulating filler via optimized process in terms of graphite size, suitable amphiphile, and processing time/steps. Generally, larger graphite/polymer composite possess higher thermal conductivity than that of smaller graphite, however, the larger graphite is less suitable to silica coated layer. The amphiphiles determine coated morphology, coating rate, growth model, and ultimately the success of the coating. The amphiphile-assisted silica@graphite that was synthesized under optimal conditions was compounded with a thermoplastic polyester elastomer (TPEE) to determine its electrically insulating properties after being subjected to shear force during high-content mixing of $50 \mathrm{wt} \%$ (100 phr). The main paragraph text follows directly on here.

\section{Experimental}

\subsection{Materials}

Five different sizes of synthetic graphite were supplied by Timcal (Switzerland): KS6 $\left(d_{50}: 3.4 \mu \mathrm{m}, d_{90}: 6.5 \mu \mathrm{m}\right) ; \operatorname{KS} 44\left(d_{50}\right.$ : $\left.18.6 \mu \mathrm{m}, d_{90}: 45.4 \mu \mathrm{m}\right) ; \operatorname{KS} 75\left(d_{50}: 23.1 \mu \mathrm{m}, d_{90}: 55.8 \mu \mathrm{m}\right) ; \mathrm{KS} 150$ $\left(d_{50}\right.$ : approximately $100 \mu \mathrm{m},{ }^{30} d_{90}$ : approximately $\left.140 \mu \mathrm{m}\right)$; and KS500 (approximately $300 \mu \mathrm{m},{ }^{30} d_{90}$ : about $550 \mu \mathrm{m}$ ). Octylphenyl 9.5 ethoxylate (Triton X-100) was provided by Samchun (S. Korea), and polyvinylpyrrolidone (PVP, $M_{\mathrm{w}}: 40000 \mathrm{~g} \mathrm{~mol}^{-1}$ ) was supplied by Junsei. Lignin (alkali), polyethyleneglycol (PEG, $M_{\mathrm{n}}$ : 400, 2050, 4600, $10000 \mathrm{~g} \mathrm{~mol}^{-1}$ ), polyethyleneimine (PEI, $M_{\mathrm{w}}$ : $800 \mathrm{~g} \mathrm{~mol}^{-1}$ ), and sodium dodecyl sulfate (SDS) were supplied by Sigma-Aldrich (USA). Tetraethyl orthosilicate (TEOS, $\geq 95 \%$ ) from Junsei Chemical Co., Ltd. (Japan), ammonium hydroxide (25 wt\% $\mathrm{NH}_{3}$ in water) from OCI Co., Ltd. (S. Korea) and ethanol from Duksan Chemicals Co., Ltd. (S. Korea) were selected.

\subsection{Sample preparation}

2.2.1. Silica coating on the graphite surface. In previous research, the silica coating process was performed in two steps: (1) the physical adsorption of PVP on the graphite surface and (2) silica coating on the PVP@graphite. ${ }^{18,19}$ In contrast, in our study, silica was coated on the graphite surface with physical adsorption of an amphiphile in one step, without two-time filtration and drying. A $5 \mathrm{~g}$ sample of graphite was poured into $75 \mathrm{ml}$ of ethanol with $0.5 \mathrm{~g}$ of an amphiphile such as Triton $\mathrm{X}-100$, PVP, lignin, SDS, PEI, or PEG. The mixtures were stirred at $500 \mathrm{rpm}$ for $3 \mathrm{~h}$. After the amphiphile was adsorbed onto the graphite, ammonium hydroxide (serving as a catalyst for the sol-gel reaction) and TEOS (acting as a silica precursor) were poured into the mixing solution in a $1: 3$ ratio. The TEOS sol- 


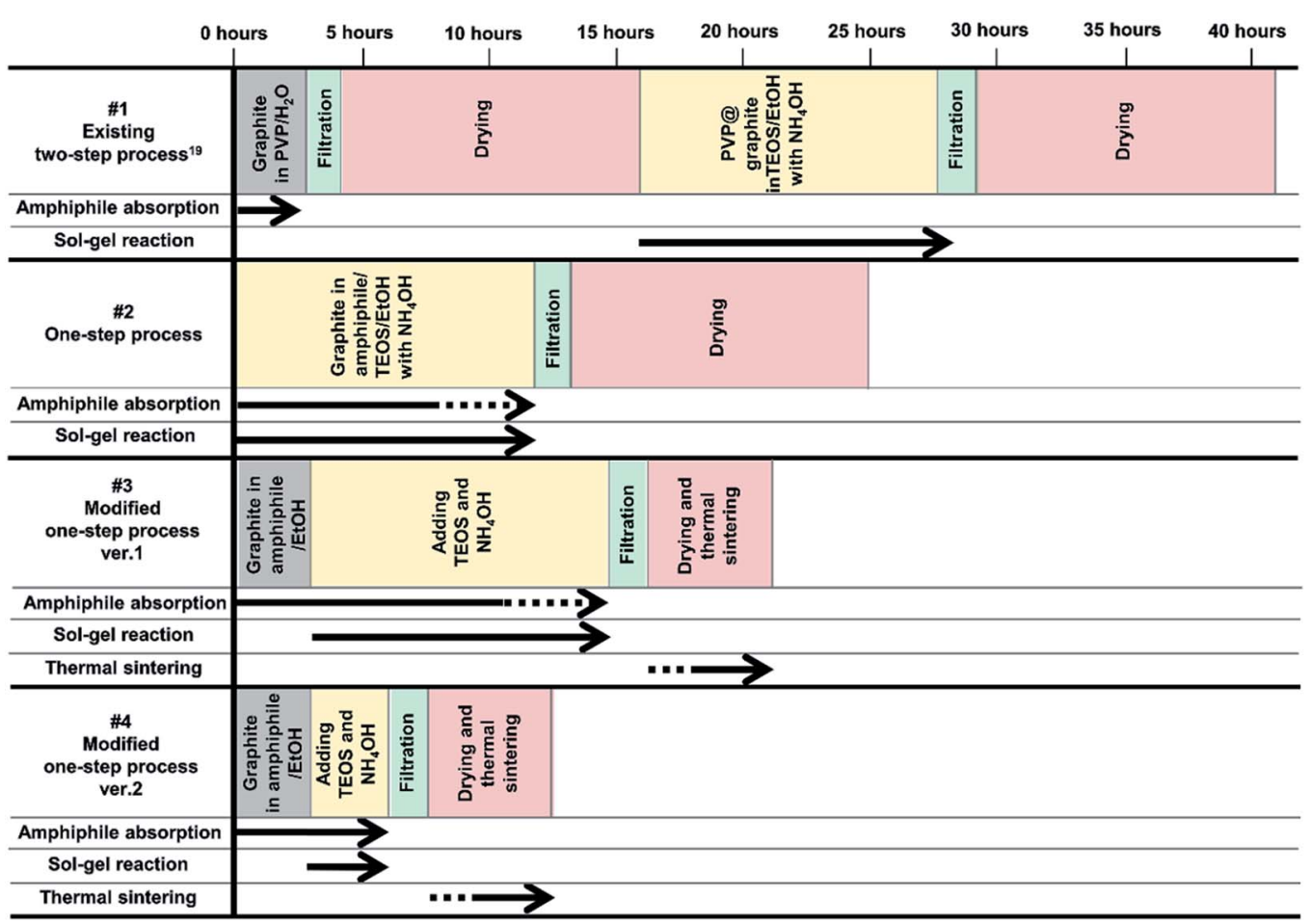

Fig. 2 Four processes (existing two-step, ${ }^{19}$ one-step, modified one-step ver. 1 and ver. 2) for synthesizing amphiphile-assisted silica@graphite.

gel reaction was stirred at room temperature for $12 \mathrm{~h}$. After coating, the mixture was filtered and washed with ethanol. Finally, the wet filler-cake was dried in an oven at $60^{\circ} \mathrm{C}$. In the thermal sintering work, the wet filler cake was sintered in a furnace at $500{ }^{\circ} \mathrm{C}$ for $5 \mathrm{~h}$. Tables 1 and 2 show the additional experimental conditions (for the experimental conditions of the one-step process and modified one-step process, ver. 2, see ESI†े).

2.2.2. Preparation of amphiphile-assisted silica@graphite/ TPEE composites. Raw graphite and amphiphile-assisted silica@graphite were incorporated into molten TPEE resin at $260{ }^{\circ} \mathrm{C}$, with a rotor speed of $100 \mathrm{rpm}$ for $10 \mathrm{~min}$. The composites were hot-pressed to obtain the desired dimensions in chrome-coated steel molds under approximately $32 \mathrm{MPa}$ at $260{ }^{\circ} \mathrm{C}$, and were cooled to $150{ }^{\circ} \mathrm{C}$ at an average cooling rate of $5{ }^{\circ} \mathrm{C} \min ^{-1}$.

\subsection{Characterization}

Field emission transmission electron microscopy (FE-TEM, CM 2000, Philips) and scanning electron microscopy (SEM, Hitachi S-4300) were used to observe the surface morphology of the coated silica on the graphite surface. Quantitative changes in the composition of the silica@graphite were calculated with thermogravimetric analysis (TGA, Diamond TG/DTA Lab System, Perkin Elmer Inc.) over a temperature range of 50$900{ }^{\circ} \mathrm{C}$ at a heating rate of $5{ }^{\circ} \mathrm{C} \mathrm{min}^{-1}$ in air. Prior to TGA, the samples were dried overnight in a vacuum oven at $60^{\circ} \mathrm{C}$. A quick thermal conductivity meter (QTM-500, Kyoto Electronics) was used to measure the thermal conductivity of the composites. The surface resistivity of the fillers and their thermoplastic polyester elastomer (TPEE) composites was measured using a high-resistivity meter (Hiresta-UP, Mitsubishi Chemical Co.) and a low-resistivity meter (Loresta-GP, Mitsubishi Chemical Co.). A high-resistivity meter uses constant-voltage processing with a concentric ring probe and a measurement range of $10^{4}$ to $10^{13} \Omega$, and a low-resistivity meter uses constant-current processing with a linear four-point probe and a measurement range of $10^{-3}$ to $10^{7} \Omega$.

\section{Results and discussion}

\subsection{Morphology of the amphiphile-assisted silica@graphite}

3.1.1. Effect of amphiphile type on coating morphology of the $12 \mu \mathrm{m}$ silica@graphite. Fig. 3 presents SEM images of the amphiphile-assisted silica@graphite synthesized using four different amphiphiles and two different combinations: Triton $\mathrm{X}-100$, PVP, lignin, SDS, Triton X-100/PVP, and SDS/PVP. The coating morphology features were analyzed using five metrics (see Fig. 3g). The diagrams represent characteristics describing aspects of the coating dispersion, which are determined by functional group characteristics and distributions. Thus, they reveal: (left side) the distribution state of the functional groups for the uniform dispersion of silica species, (right side) the functional group characteristics that facilitate the growth of silica species, and (top) the concomitant interaction resulting from the growth routes (i.e., the growth tendencies of the Volmer-Weber mode, Frank-van der Merwe mode, step flow mode; Stranski-Kranstranov mode; and columnar growth mode $^{31}$ ) (details of the five metrics are available in ESI $\dagger$ ). The size of the pentagon indicates the level of each metrics. The 
Table 1 Experimental conditions for physical adsorption of amphiphile and silica coating on graphite $\left(d_{50}: 12 \mu \mathrm{m}\right)$

\begin{tabular}{|c|c|c|c|c|c|c|c|c|c|}
\hline \multirow[b]{2}{*}{ No. } & \multicolumn{2}{|c|}{ Graphite } & \multicolumn{2}{|c|}{ Amphiphile } & \multirow{2}{*}{$\begin{array}{l}\mathrm{NH}_{4} \mathrm{OH} \\
\text { Mass ratio } \\
\text { of } \mathrm{NH}_{4} \mathrm{OH} \\
\text { to TEOS }\end{array}$} & \multicolumn{2}{|l|}{ TEOS } & \multirow{2}{*}{$\begin{array}{l}\text { Media } \\
\text { Volume (ml) } \\
\text { of EtOH }\end{array}$} & \multirow{2}{*}{$\begin{array}{l}\text { Reaction time } \\
\text { Time (h) }\end{array}$} \\
\hline & $\begin{array}{l}d_{50} / d_{90} \\
(\mu \mathrm{m})\end{array}$ & $\begin{array}{l}\text { Mass } \\
(\mathrm{g})\end{array}$ & $\begin{array}{l}\text { Mass } \\
(\mathrm{g})\end{array}$ & $\begin{array}{l}\text { Mass } \% \text { of } \\
\text { amphiphile input } \\
\text { compared with graphite }\end{array}$ & & $\begin{array}{l}\text { Mass }(g) \text { of } \\
1^{\text {st }} / 2^{\text {nd }} \text { input }\end{array}$ & $\begin{array}{l}\text { Mass } \% \text { of } \\
\text { amphiphile input } \\
\text { compared with graphite }\end{array}$ & & \\
\hline $1-6$ & $12 / 25^{a}$ & 5 & $0.50^{b}$ & 10 & $1: 3$ & $6.0 / 0$ & $120 / 0$ & 75 & 12 \\
\hline \multirow[t]{7}{*}{$7-18$} & $12 / 25^{a}$ & 5 & $0.25^{c}$ & 5 & $1: 3$ & $1.0 / 0$ & $20 / 0$ & 75 & 12 \\
\hline & & & $0.25^{c}$ & 5 & & $6.0 / 0$ & $120 / 0$ & & \\
\hline & & & $0.50^{c}$ & 10 & & $1.0 / 0$ & $20 / 0$ & & \\
\hline & & & $0.50^{c}$ & 10 & & $2.0 / 0$ & $40 / 0$ & & \\
\hline & & & $0.50^{c}$ & 10 & & $4.0 / 0$ & $80 / 0$ & & \\
\hline & & & $0.50^{c}$ & 10 & & $6.0 / 0$ & $120 / 0$ & & \\
\hline & & & $2.00^{c}$ & 40 & & $1.0 / 0$ & $20 / 0$ & & \\
\hline & & & & & & & & & 6 \\
\hline & & & & & & & & & 9 \\
\hline & & & & & & & & & 10 \\
\hline & & & & & & & & & 11 \\
\hline & & & & & & & & & 12 \\
\hline
\end{tabular}

${ }^{a}$ Two different size of raw graphite, KS6 $\left(d_{50} 3.4 \mu \mathrm{m}, d_{90} 6.5 \mu \mathrm{m}\right)$ and KS44 $\left(d_{50} 18.6 \mu \mathrm{m}, d_{90} 45.4 \mu \mathrm{m}\right)$, were prepared with a mass ratio of $1: 1 .{ }^{b}$ The amphiphiles used in the preparation of samples \#1-6 were: (1) Triton X-100, (2) PVP, (3) lignin, (4) SDS, (5) a hybrid of Triton X-100 and PVP with a mass ratio of $1: 1,(6)$ a hybrid of SDS and PVP with a mass ratio of $1: 1$. $^{c}$ The amphiphile used in the preparation of samples \#7-24 was Triton-X 100.

inner side of the pentagon is suitable for yielding smooth and compact coverage. The middle 3rd pentagon is the Maginot line-full coverage with a surface resistivity of $10^{10}$ to $10^{13} \mathrm{ohm}$ $\mathrm{sq}^{-1}$ was attained, and 5 angular points were located in the area of the middle 3rd pentagon. Fig. 3a (Triton X-100) and Fig. 3e (Triton X-100/PVP) agree with the 3rd line; they show a smooth

Table 2 Experimental conditions for physical adsorption of amphiphile and silica coating on graphite $\left(d_{50}: 12 / 23 / 100 / 300 \mu m\right)$

\begin{tabular}{|c|c|c|c|c|c|c|c|c|c|}
\hline \multirow[b]{2}{*}{ No. } & \multicolumn{2}{|c|}{ Graphite } & \multicolumn{2}{|c|}{ Amphiphile } & \multirow{2}{*}{$\begin{array}{l}\mathrm{NH}_{4} \mathrm{OH} \\
\text { Mass ratio } \\
\text { of } \mathrm{NH}_{4} \mathrm{OH} \\
\text { to TEOS }\end{array}$} & \multicolumn{2}{|l|}{ TEOS } & \multirow{2}{*}{$\begin{array}{l}\text { Media } \\
\text { Volume (ml) } \\
\text { of EtOH }\end{array}$} & \multirow{2}{*}{$\begin{array}{l}\text { Reaction time } \\
\text { Time (h) }\end{array}$} \\
\hline & $\begin{array}{l}d_{50} / d_{90} \\
(\mu \mathrm{m})\end{array}$ & $\begin{array}{l}\text { Mass } \\
(\mathrm{g})\end{array}$ & $\begin{array}{l}\text { Mass } \\
(\mathrm{g})\end{array}$ & $\begin{array}{l}\text { Mass } \% \text { of } \\
\text { amphiphile input } \\
\text { compared with graphite }\end{array}$ & & $\begin{array}{l}\text { Mass (g) of } \\
1^{\text {st }} / 2^{\text {nd }} \text { input }\end{array}$ & $\begin{array}{l}\text { Mass } \% \text { of } \\
\text { amphiphile input } \\
\text { compared with graphite }\end{array}$ & & \\
\hline $25-30$ & $100 / 140$ & 5 & $0.50^{b}$ & 10 & $1: 3$ & $2.7 / 1.3$ & $53 / 27$ & 75 & 12 \\
\hline \multirow[t]{9}{*}{$31-39$} & $100 / 140$ & 5 & $0.25^{c}$ & 5 & $1: 3$ & $0.7 / 0.3$ & $7 / 13$ & 75 & 12 \\
\hline & & & $0.25^{c}$ & 5 & & $1.3 / 0.7$ & $27 / 13$ & & \\
\hline & & & $0.25^{c}$ & 5 & & $2.7 / 1.3$ & $53 / 27$ & & \\
\hline & & & $0.50^{c}$ & 10 & & $0.7 / 0.3$ & $7 / 13$ & & \\
\hline & & & $0.50^{c}$ & 10 & & $1.3 / 0.7$ & $27 / 13$ & & \\
\hline & & & $0.50^{c}$ & 10 & & $2.7 / 1.3$ & $53 / 27$ & & \\
\hline & & & $1.00^{c}$ & 20 & & $0.7 / 0.3$ & $7 / 13$ & & \\
\hline & & & $1.00^{c}$ & 20 & & $1.3 / 0.7$ & $27 / 13$ & & \\
\hline & & & $1.00^{c}$ & 20 & & $2.7 / 1.3$ & $53 / 27$ & & \\
\hline \multirow[t]{8}{*}{$40-59$} & $12 / 25^{a}$ & 5 & $0.5^{d}$ & 10 & $1: 3$ & $2.7 / 1.3$ & $53 / 27$ & 75 & 12 \\
\hline & $23 / 55$ & & $0.5^{d}$ & 10 & & & & & \\
\hline & $100 / 140$ & & $0.5^{d}$ & 10 & & & & & \\
\hline & $300 / 550$ & & $0.5^{d}$ & 10 & & & & & \\
\hline & $12 / 25^{a}$ & & None & 0 & & & & & \\
\hline & $23 / 55$ & & None & 0 & & & & & \\
\hline & $100 / 140$ & & None & 0 & & & & & \\
\hline & $300 / 550$ & & None & 0 & & & & & \\
\hline
\end{tabular}

${ }^{a}$ Two different size of raw graphite, KS6 $\left(d_{50} 3.4 \mu \mathrm{m}, d_{90} 6.5 \mu \mathrm{m}\right)$ and KS44 $\left(d_{50} 18.6 \mu \mathrm{m}, d_{90} 45.4 \mu \mathrm{m}\right)$, were used with a mass ratio of $1: 1 .^{b}$ The amphiphiles used for samples \#25-30 were: (25) Triton X-100, (26) PVP, (27) lignin, (28) SDS, (29) PEI, and (30) PEG ( $M_{\mathrm{n}} 4600 \mathrm{~g}^{\mathrm{mol}}{ }^{-1}$ ). ${ }^{c} \mathrm{The}$ amphiphile used for samples \#31-39 was a PEG of $M_{\mathrm{n}} 4600 \mathrm{~g} \mathrm{~mol}^{-1}$. ${ }^{d}$ Molecular weights $\left(M_{\mathrm{n}}\right)$ of PEG for samples \#40-55 were (40-43) 400, (44-47) 2060, (48-51) 4600, and (52-55) $10000 \mathrm{~g} \mathrm{~mol}^{-1}$. 

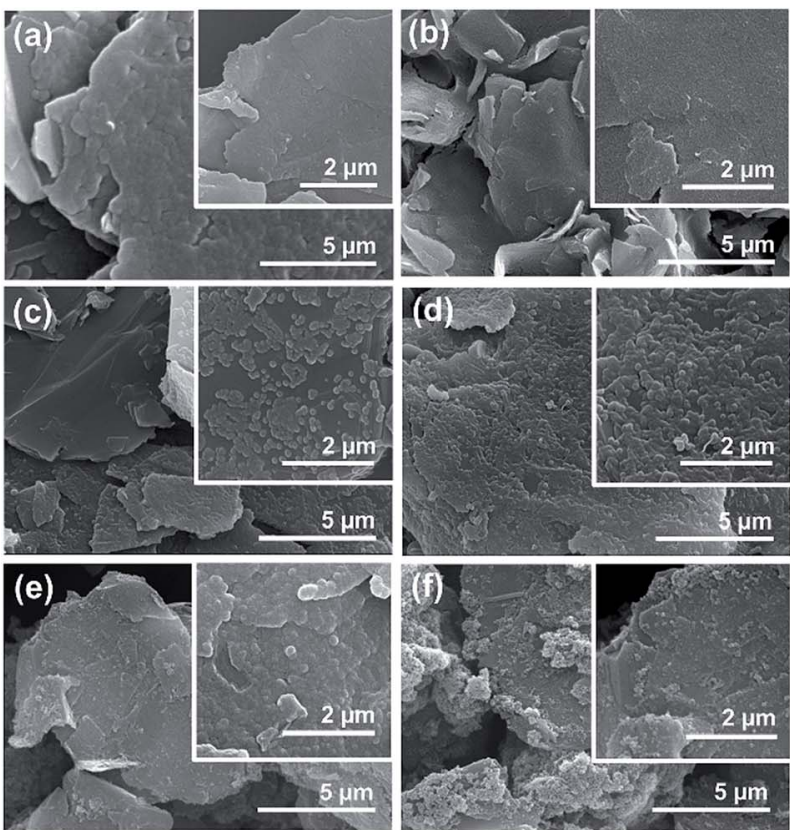

(g)

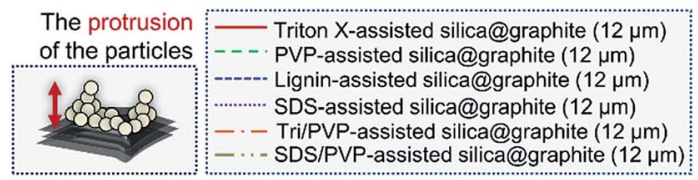

The average size and frequency of empty area $\mathrm{b} / \mathrm{n}$ the particles

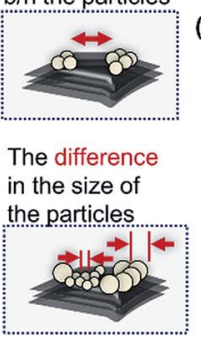

(empty)

(Protrusive)

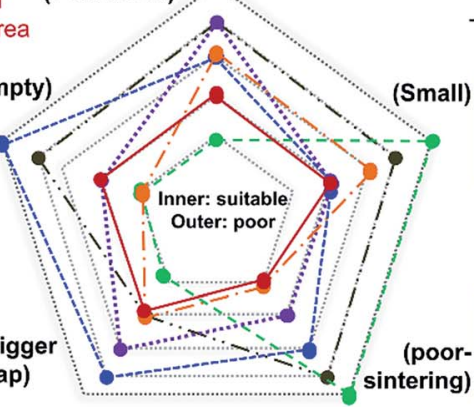

The average size of the particles ...........................
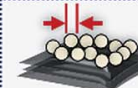

The progress of sintering $\mathrm{b} / \mathrm{n}$ the particles

\begin{tabular}{|c|c|c|c|c|c|c|}
\hline & Triton $\mathrm{X}$ & PVP & Lignin & SDS & Tri./PVP & SDS/PVP \\
\hline $\begin{array}{c}\text { Area } \\
\text { on the } \\
\text { graph }\end{array}$ & N & & & & & \\
\hline
\end{tabular}

Fig. 3 FE-SEM images of silica@graphite surfaces $\left(d_{50} 12 \mu \mathrm{m}, d_{90} 25 \mu \mathrm{m}\right)$ prepared using (a) Triton-X, (b) PVP, (c) lignin, (d) SDS, (e) combination of Triton-X/PVP, (f) combination of SDS/PVP, and (g) morphology analysis.

and compact coating-surface with approximately full coverage. The filling of the few channels on the basal plane of Fig. 3a (Triton X-100) can be supported by the seed-nuclei, which prevail on the graphite surface (Fig. 3b (PVP)). The synergy of the combination of Triton X-100/PVP can be observed in Fig. 3e. In Fig. $3 \mathrm{~b}$ (PVP), although the nuclei spread out over the entire area and have superior distribution, the growth of the nuclei was unsuccessful. Islands can form on the edge of the graphite (average $12 \mu \mathrm{m}$ ) without an amphiphile, because of the high surface energy and functional groups of the edge ${ }^{18}$ (for information on the micrograph and surface resistivity of the silica@graphite in the absence of amphiphiles, see ESI $\dagger$ ). On the other hand, no islands were observed on the edge of the PVPassisted silica@graphite (Fig. 3b (PVP)). Therefore, there are insufficient active sites on the covered PVP to facilitate the growth of silica, and the PVP covering disturbs the edge growth of the silica species. Fig. 3c (lignin) shows the channels resulting from an uneven distribution of hydroxyl groups. In Fig. 3d (SDS), the rough layer and protruding particles cover the graphite surface, without creating large channels. A comparison of Fig. 3a (Triton X-100) with Fig. 3d (SDS) shows that Triton X100 provides more functional groups per unit area of graphite than SDS. In contrast to that depicted in Fig. 3e (Triton X-100/ PVP), the morphology of Fig. 3f (SDS/PVP) falls under the overriding influence of PVP. Consequentially, Triton X-100 was selected as a suitable amphiphile for the silica coating of $12 \mu \mathrm{m}$ graphite.

Fig. 4 shows SEM images of Triton X-assisted silica@graphite synthesized with two different variables: the amounts of Triton X-100 and TEOS. The optimal conditions for uniform silica coating on graphite were found to be $10 \mathrm{wt} \%$ of Triton X100 and $120 \mathrm{wt} \%$ of TEOS with respect to the mass of graphite.

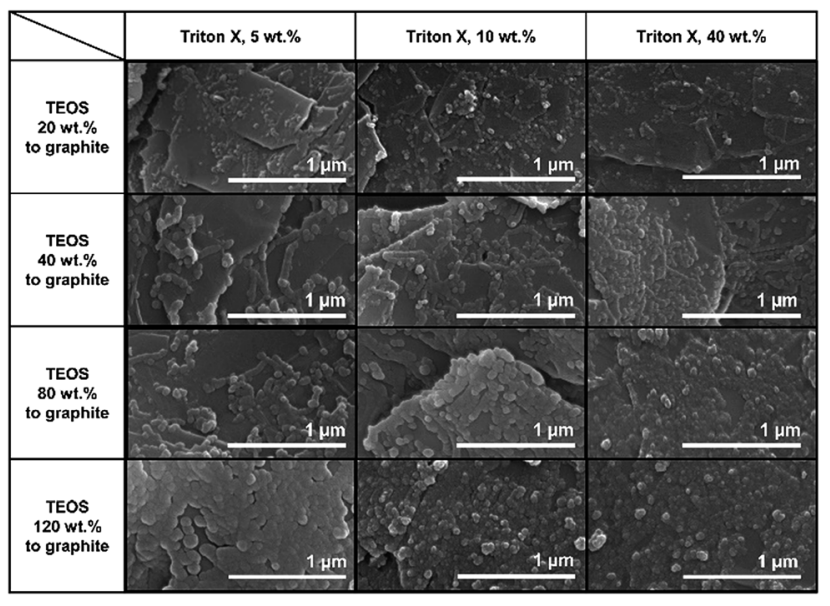

Fig. 4 FE-SEM images of the surfaces of the Triton $X$-assisted silicalagraphite $\left(d_{50} 12 \mu \mathrm{m}, d_{90} 25 \mu \mathrm{m}\right)$ synthesized with different amounts of Triton X-100 and TEOS.

Three facts regarding the silica-coating mechanism can be deduced from the coating tendencies. The formation and growth of nuclei occurs preferentially on the edge rather than the basal plane of graphite. The growth of nuclei tends to proceed via the connection of grown particles and absorption of adjacent embryos. In other words, few islands are separated from the grown silica species that are based on the edge. Triton $\mathrm{X}-100$ helps the silica species grow toward the basal plane and fills the many channels with silica species.

3.1.2. Effects of amphiphile type on coating morphology of the $100 \mu \mathrm{m}$ silica@graphite. The contribution of the amphiphile to the basal plane of $100 \mu \mathrm{m}$ graphite (specific surface area: $3 \mathrm{~m}^{2} \mathrm{~g}^{-1}$, BET) far outweighs that of the $12 \mu \mathrm{m}$ graphite 
(specific surface area: $17.5 \mathrm{~m}^{2} \mathrm{~g}^{-1}$, BET). The strategy, which depends on the growth of silica species on the edge, is too inefficient to coat the $100 \mu \mathrm{m}$ graphite, because of the large basal plane and small edge. Therefore, the formation of a silica layer on the large graphite surface (over $100 \mu \mathrm{m}$ ) requires an amphiphile that provides a basal plane with sufficient hydroxyl groups.

Fig. 5 shows SEM images of silica@graphite synthesized with six different amphiphiles: Triton X-100, PVP, lignin, SDS, PEI, and PEG. The coating morphology was analyzed with five metrics, as shown in Fig. 5g. An item that abides by the 3rd line is shown only in Fig. 5e (PEG), which shows a smooth and compact coating-surface with full coverage, containing few cracks on the thick silica layer. In contrast to what is shown for the $12 \mu \mathrm{m}$ Trion X-assisted silica@graphite, the growth of nuclei is suppressed, and there are no large particles to absorb embryos (see Fig. 5a, Triton X-100). Triton X-100 is a subsidiary amphiphile, which leads silica species to grow from the edge to the basal plane. Triton $\mathrm{X}-100$ is unable to facilitate growth toward the $Z$-axis without large adjacent silica species. Therefore, the growth of the silica species on the middle portions of the wide basal plane in this system is not expected. Hong et al. suggested that a liquid crystal surfactant/silicate film is aligned with a hemimicelle-graphite structure. Micelles of cetyltrimethylammonium chloride (CTACl) were assembled in an aqueous medium under acidic conditions; the silica species grew from the outer hydrophilic heads of the micelles, which were stacked in tiers. The behavior facilitated growth toward the $Z$-axis. ${ }^{24}$ On the other hand, the existence of micelles is rare in an alcoholic medium containing approximately 5-10\% water (25\% ammonia solution and 95\% ethanol were employed) under basic conditions. In the cases shown in Fig. 5b (PVP), Fig. 5c (lignin), Fig. 5d (SDS), and Fig. 5f (PEI), there were insufficient seed nuclei, meaning that there was a lack of amphiphile adsorption on the graphite. Four characteristics regarding the silica-coating mechanism can be deduced from the coating tendencies. (1) The hydrophobic portion of Triton X100 binds to graphite more easily than that of SDS. (2) Triton X100 cannot form islands with the silica species on the wide basal plane (graphite over $100 \mu \mathrm{m}$ ). (3) The PEG backbone has the advantage of physical adsorption onto graphite compared to PVP and PEI backbones. (4) In this system, lignin is an indefinite and vague amphiphile, with weak physical adsorption and uneven hydroxyl groups. As a result, PEG was selected as a suitable amphiphile for a silica coating of the $100 \mu \mathrm{m}$ graphite.

3.1.3. Effect of sol-gel reaction time on the coating morphology of the amphiphile-assisted silica@graphite. Growth models and optimal sol-gel reaction times were obtained from changes in morphology as a function of the solgel reaction time (Fig. 6a). The growth of silica species for Triton X-assisted silica@graphite is categorized by the Volmer-Weber growth model, which shows typical island growth. Accordingly, the sol-gel reaction time for full coverage depends on the time to form nuclei on the functional groups of the edge and adjacent Triton X-100 (0-3 h), the before a critical size with stable free energy is reached (6-9 h), and time for grown nuclei to fill the channels on the basal, supported by Triton X-100 (12 h). The entire sol-gel reaction procedure with Triton $\mathrm{X}-100$ requires $12 \mathrm{~h}$ for reliable and reproducible full coverage. The growth of the silica species for the PEG-assisted silica@graphite in Fig. 6b shows tendencies of a Frank-van der Merwe growth model/Volmer-Weber growth model hybrid. (1) Active and small silica species dispersed in a medium are bound to the hydroxyl groups located at both terminals of the PEG chains on the graphite. In
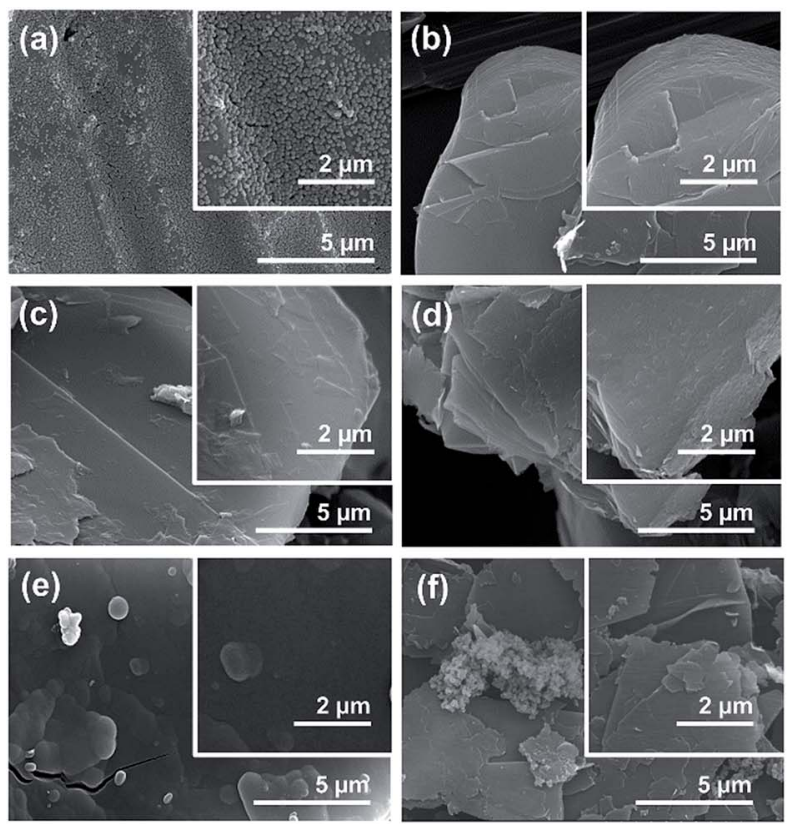

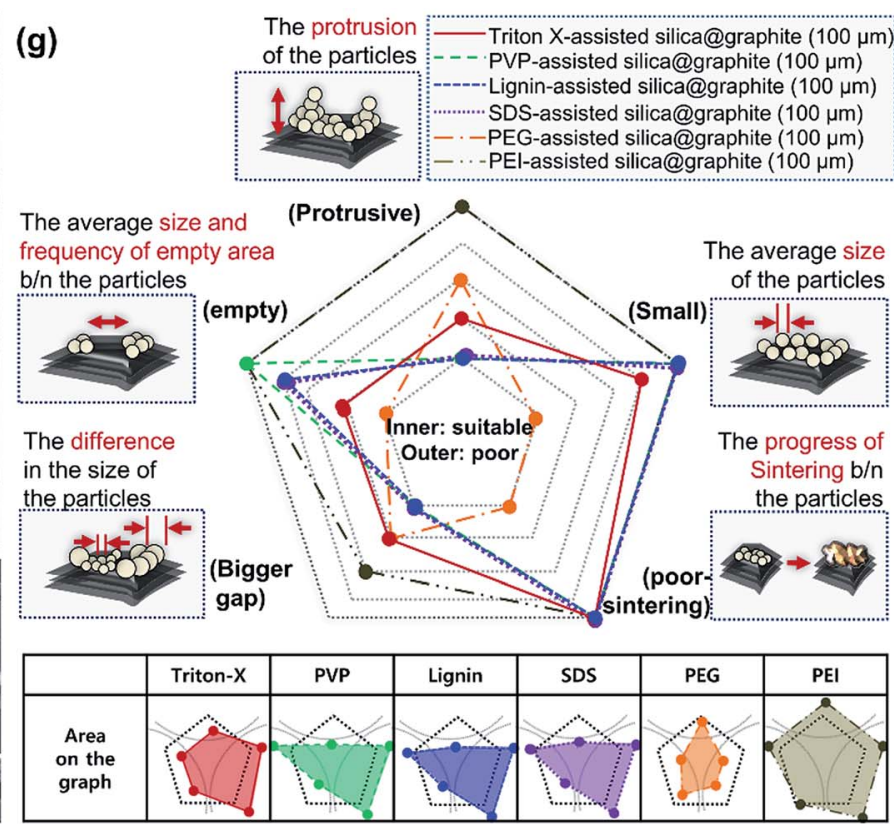

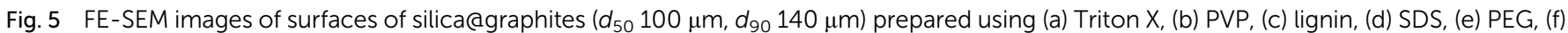
$\mathrm{PEI}$, and (g) morphology analysis. 
(a)
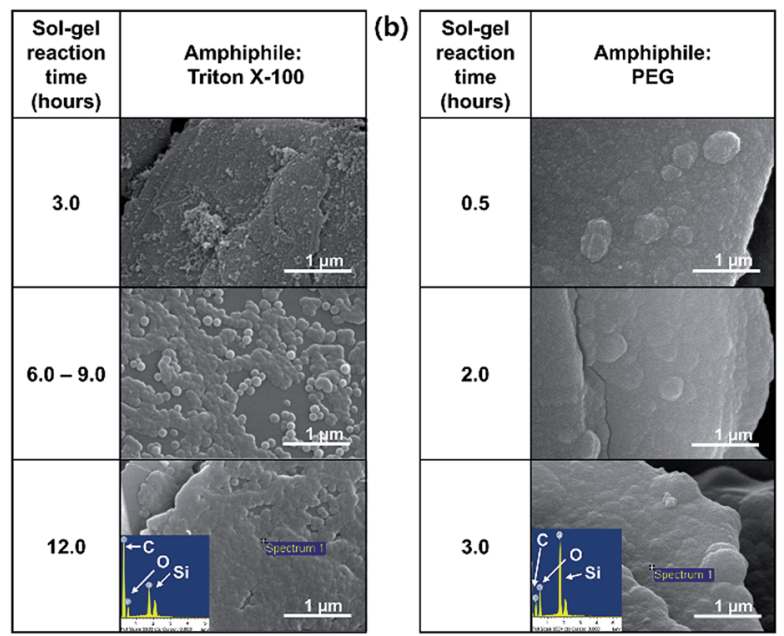

Fig. 6 FE-SEM images of surfaces of (a) the Triton X-assisted silica@graphite $(12 \mu \mathrm{m})$ and (b) PEG-assisted silica@graphite $(100 \mu \mathrm{m})$ depending on the sol-gel reaction time.

addition, growing silica species dispersed in a medium are bound randomly to a thin layer without a preponderance of area (0-2 h). (2) Grown silica species dispersed randomly in the medium are bound to the growing layer without a preponderance of area. At the same time, the silica layer grows, with a tendency to even-out the gap between the protruding surface and adjacent irregular surface $(3 \mathrm{~h})$. Based on the diversification of growth routes and growing points, the entire sol-gel reaction procedure for PEG requires $3 \mathrm{~h}$, which is remarkably shortened to one-fourth of the original required time (for EDAX data, ESI $\dagger$ ).

3.1.4. Effects of amphiphile and graphite size on the mechanism of silica coating on graphite. To elucidate the organizational correlation between silica-coating morphology and an amphiphile, Fig. 7 shows the molecular structures of the amphiphile, the features involved in adsorption on graphite, the hydroxyl groups for the growth of silica species, and the morphology resulting from the features. The amphiphile is categorized into groups, such as surfactants, polymers, and their hybrids.

The mechanism for surfactant (amphiphile)-assisted silica coating involves (1) the molecular structures of the surfactants, and (2) hydrophile(polar)-lipophile(non-polar) balance (HLB), given the weak VDW interaction resulting from very low molecular weight. The HLB values of Triton-X and SDS were calculated using the following equation. ${ }^{32}$

$\mathrm{HLB}=\sum$ (hydrophilic group number $)-n$ (group number per $\mathrm{CH}_{2}$ group) +7

The SDS molecule has an HLB value of 40; it incorporates a linear carbon chain on its hydrophobic portion and a sulfate on its hydrophilic portion. Triton X-100 combines a linear carbon chain and a benzene ring on the hydrophobic portion with a long chain of polyoxyethylene on the hydrophilic portion. Although the molecule has a moderate HLB value of 13.5, its hydrophobic portion allows it to closely approach graphite. Just as "like dissolves like" in the world of solvents, the concept of "like attracts like" is considered in this system in terms of how the hydrophobic portion of the surfactant interacts with graphite. Compared to the HLB and molecular structure of SDS, Triton X-100 is more suitable for binding to graphite because of the more hydrophobic HLB and benzene ring. Because of the more bounded Triton X-100, Triton X-100@graphite can provide a larger number of hydrophilic-functional groups on graphite than SDS@graphite in the system for the same reaction time. This forms more seed-nuclei on the graphite than in the case of SDS with the same reaction time. In addition, the more seed-nuclei also facilitates the overlap of silica specieshemispheres (nuclei and island) on graphite. Consequently, Triton X-assisted silica@graphite has a smoother and more compact coating-surface than SDS-assisted silica@graphite. The amphiphile of the surfactant type (i.e., Triton X-100 and SDS) provides nuclei that are surrounded by functional groups forming embryos; the amphiphile facilitates the adsorption of embryos by the nuclei. Proceeding further, such behavior assists the formation of islands and nuclei layers via Oswald ripening. Therefore, amphiphiles are referred to as "Oswald ripening agents".

The mechanism of the polymer (amphiphile)-assisted silica coating is under a dominant influence on the VDW interaction that resulting from the high molecular weight and the structures of the polymers. As mentioned in Section 3.1.1, the PVP covering disturbs the edge growth of silica species, which means that the PVP chains are firmly bound to the edge. In the case of surfactants, such as SDS and Triton X-100, the hydrophobic portions of the surfactants can also bind to edges, but no channels are observed on the edge of the surfactantassisted silica@graphite. Three characteristics of this mechanism can be deduced from the results. The surfactants can escape the edge and adsorb to the basal plane. The physical adsorption of polymers by VDW interactions enables them to firmly bind to all areas (basal plane and edge) of the graphite. Therefore, polymer-assisted silica coating cannot support the edge growth of silica species in this system. In terms of the molecular structures of the polymers, hydroxyl groups are located on: the end of the pyrrolidone rings of the PVP enoltautomer backbone, ${ }^{19}$ the irregular points of the lignin network that is intertwined with diverse functional groups, and the PEG terminals. Accordingly, in comparison to PVP and lignin backbone chains, PEG backbone chains can (advantageously) physically adsorb onto graphite. The steric hindrance that results from the pyrrolidone rings of the PVP enol tautomer prevents the hydroxyl groups from encountering the silanol group of the silica precursor molecule. Moreover, the keto-enol tautomerism of PVP is a reversible reaction under basic conditions; the PVP layer on graphite cannot always provide stable hydroxyl groups. Although a PEG chain has just two hydroxyl groups on both terminals, and the total number of hydroxyl groups per volume is lower than that of other amphiphiles, PEG chains can form 'silica sol-PEG' networks as cross-linking of polymers. ${ }^{33}$ Therefore, the 'silica sol-PEG' network reacts on (route 1) the hydroxyl groups of PEG chains adsorbed to the graphite, (route 2) the hydroxyl groups of PEG 

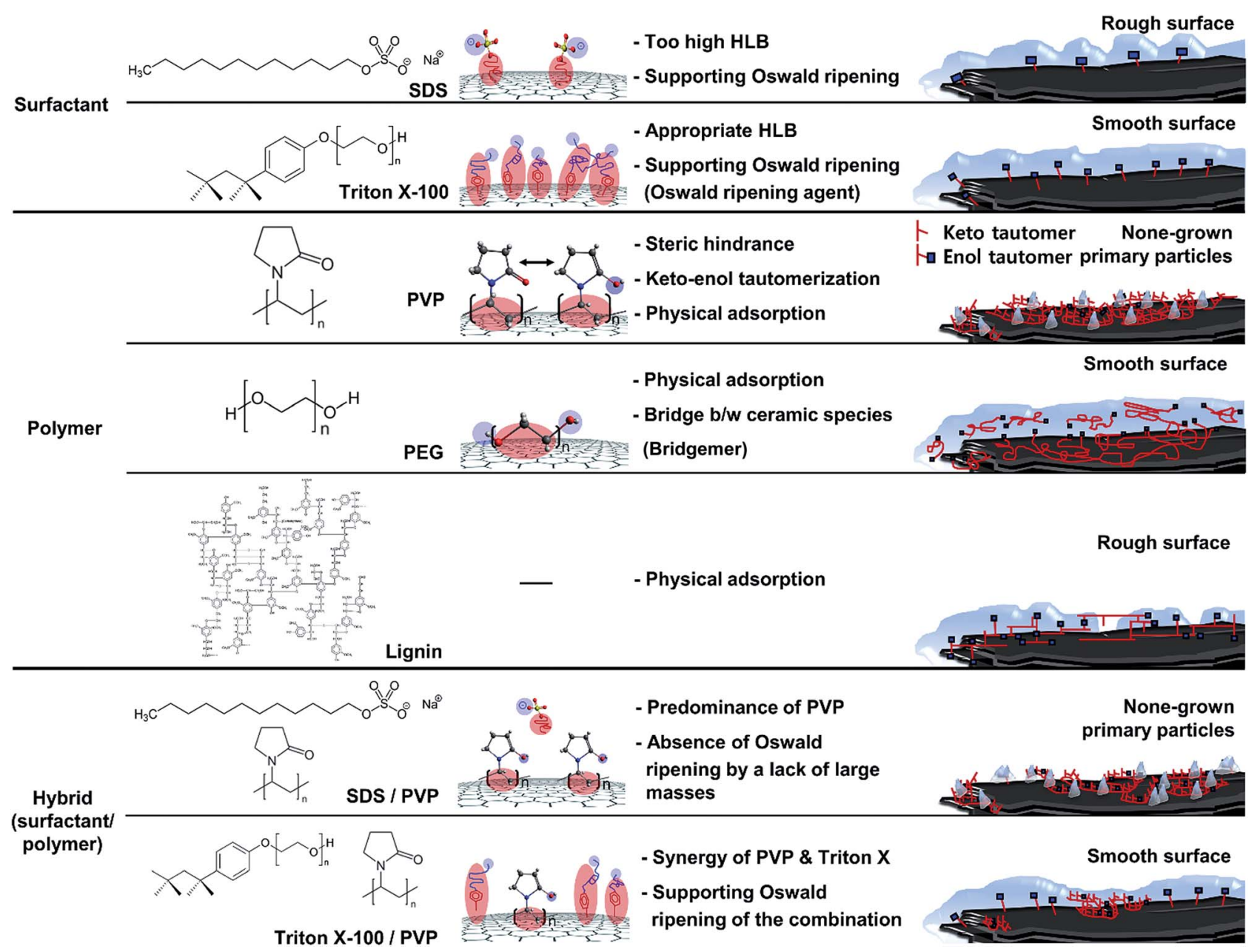

Fig. 7 Mechanism of silica coating on graphite depending on amphiphile.

bound on silica species (i.e., nuclei, islands) on the graphite, and (route 3) the silanol groups of silica species (i.e., nuclei, islands) condensed on the graphite. Based on these routes, PEG facilitates the individual growth of silica species toward the $Z$-axis, without adjacent nuclei and islands on the basal plane and edges. Therefore, an amphiphile that (1) couples the sol masses and (2) binds the networks to the other material substrate can be called a "bridgemer". As a result, PEGassisted silica@graphite has a smoother and more compact coating-surface, while the nuclei of the PVP-assisted silica@graphite cannot grow into islands. Small $(12 \mu \mathrm{m})$ ligninassisted silica@graphite has large channels resulting from irregular islands and nuclei. Large $(100 \mu \mathrm{m})$ lignin-assisted silica@graphite has no islands; the nuclei are few and minuscule.

With respect to the mechanism for a surfactant/polymer hybrid, the important qualities are its synergy effect and adverse effect, and a more-dominant amphiphile to bind it to the graphite. Although PVP cannot facilitate the growth of silica species on graphite, it provides $12 \mu \mathrm{m}$ graphite in the system with functional groups for seed-nuclei. Few channels on the coating surface of the Triton X-assisted silica@graphite are filled with silica species through the assistance of seed-nuclei resulting from PVP, whereas SDS-assisted silica@graphite loses its rough surface, and therefore loses graphite edge space because of the physical adsorption of PVP chains. The difference in adsorption on graphite is due to their molecular structure and HLB. Triton X-100, which has an HLB of 13.5, can better bind to graphite than SDS, which has an HLB of 40. There are three explanations for this: (1) the Triton-X/PVP hybrid generates a synergy effect. Although Triton-X can escape from graphite, Triton X-100 disturbs the physical adsorption of the PVP chains, particularly on the edge of the graphite. (2) The SDS/PVP hybrid generates an adverse effect. The PVP chains bind dominantly to the graphite relative to SDS, and the PVP that is bound on the edges restrains the growth of silica species there. (3) The contribution of surfactants in the surfactant/ polymer hybrid system depends on their molecular structure and HLB. Considering the interaction between surfactants and polymers from another point of view, some studies have described ceramic hollow microspheres using a pear-necklace SDS/PVP model, a core-shell model of SDS/polyacrylic acid (PAA), and a core-shell model of sodium dodecyl benzenesulfonate (SDBS)/PAA. ${ }^{27,34}$ On the other hand, the models depend on the micelle structures in an aqueous system. Consequently, the Triton-X/PVP-assisted silica@graphite has a more 
(a) Nucleation on functional groups of edge

Growth: Condensation and coalescence

Hydrolysis of TEOS

$\rightarrow \mathrm{Si}\left(\mathrm{OC}_{2} \mathrm{H}_{5}\right)_{3}(\mathrm{OH})+\mathrm{C}_{2} \mathrm{H}_{5} \mathrm{OH}$

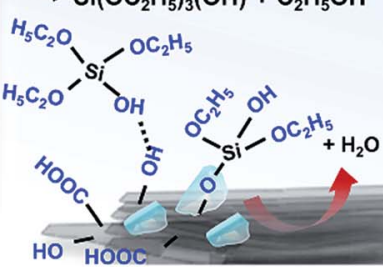

$\stackrel{5}{S}, \mathrm{OC}_{2} \mathrm{H}_{5} \longrightarrow+\mathrm{H}_{2} \mathrm{O}$<smiles>COCC=CCO[Si](C)(OC)OC</smiles>

$\mathrm{H}_{5} \mathrm{C}_{2} \mathrm{O}-\mathrm{O}$ i<smiles>CO[Si](C)(C)O[Si](C)(C)O[Si](C)(C)O</smiles>

$\rightarrow$
Imperfect silica layer

Silica coating area

resulting from

functional group on edge

(b)

\section{Nucleation on \\ functional groups of edge \\ Hydrolysis of TEOS}

$\rightarrow \mathrm{Si}\left(\mathrm{OC}_{2} \mathrm{H}_{5}\right)_{3}(\mathrm{OH})+\mathrm{C}_{2} \mathrm{H}_{5} \mathrm{OH}$

$\mathrm{H}_{5} \mathrm{C}_{2} \mathrm{O}$

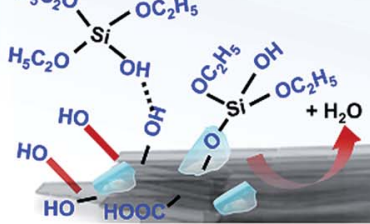

Growth: Condensation and coalescence

supported by the Oswald ripening agent
Uniform silica layer

The main supporting area by Oswald ripening agent

(c) Nucleation on functional groups of edge and terminals of PEG chains

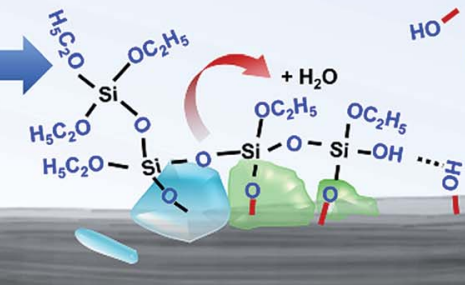

$\mathrm{OC}_{2} \mathrm{H}_{5}$ HO $1 \mathrm{OC}_{2} \mathrm{H}_{5}, \mathrm{OC}_{2} \mathrm{H}_{5}$

Channel

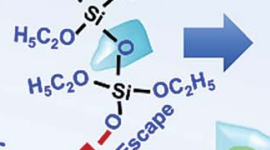

Hydrolysis of TEOS

$\rightarrow \mathrm{Si}\left(\mathrm{OC}_{2} \mathrm{H}_{5}\right)_{3}(\mathrm{OH})+\mathrm{C}_{2} \mathrm{H}_{5} \mathrm{OH}$

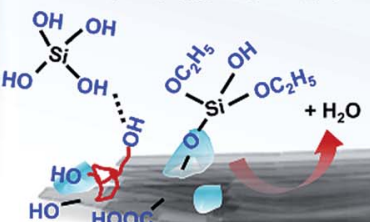
Growth: Cond
supported by
the bridgemer

1

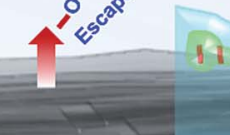

\section{(1)}

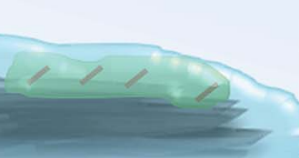

(d)

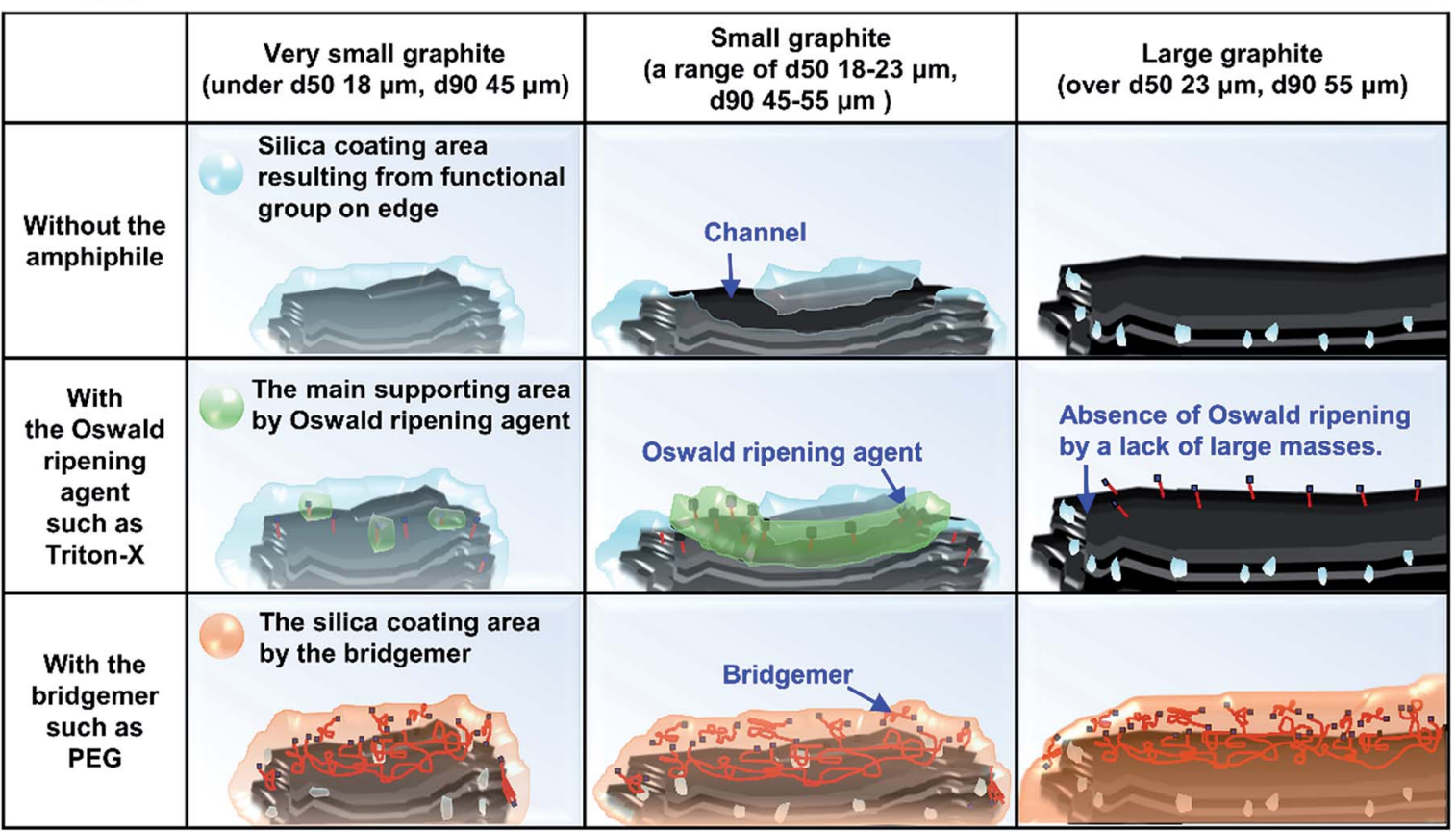

Fig. 8 Mechanism of silica coating on graphite (a) in the absence of an amphiphile, and with the assistance of (b) an Oswald ripening agent, (c) a bridgemer, and (d) amphiphile strategies for silica coating depending on the size of the graphite. 
complementary full-coverage coating-surface than that of the SDS/PVP-assisted silica@graphite.

In summary, Sections 3.1.1-3.1.4, and Fig. 8 present the mechanism of silica species growth on graphite via (a) a nonamphiphile, (b) an Oswald ripening agent such as Triton-X, (c) a bridgemer, such as PEG, and (d) three strategies depending on the graphite size.

\subsection{Surface resistivity of the amphiphile-assisted silica@graphite}

3.2.1. Effects of amphiphile and graphite size on surface resistivity of silica@graphite. According to the underlying background, which endows graphite with electrically insulating properties via a silica coating, the surface resistivity of the amphiphile-assisted silica@graphite should depend on its morphology, particularly whether the layer possesses full coverage without channels.

From a broad point of view, Fig. 9 provides information on the most suitable amphiphile for an insulating graphite treatment $\left(d_{50}\right.$ sizes of $12,23,100$, and $\left.300 \mu \mathrm{m}\right)$, and the inextricable correlation between surface resistivity and morphology. Fig. 9a shows the surface resistivity of an amphiphile-assisted silica@graphite (with sufficient TEOS applied for full coverage) synthesized with two different variables: the $d_{50}$ size of the graphite and amphiphile. In contrast to the $2.13 \times 10^{12} \mathrm{ohm}$ $\mathrm{sq}^{-1}$ of PVP-assisted silica@graphite $\left(d_{50}\right.$ size $\left.=12 \mu \mathrm{m}\right)$ that was prepared via a two-step process ${ }^{18,19}$ the PVP-assisted silica@graphite resulting from the modified one-step process maintains low surface resistivity, in the range of $10^{2} \mathrm{ohm} \mathrm{sq}^{-1}$ over all sizes of employed graphite. The additional physical adsorption work, which entails a drying time of $12 \mathrm{~h}$, enables the PVP chains to adequately bind to the surface of the graphite. Additionally, the enol tautomer of the PVP, which is bound on the graphite via the two-step process, provides sufficient hydroxyl groups. The two-step process improves the physical absorption of PVP relative to the one-step process. Nevertheless, the PVPassisted two-step process is not capable of coating a graphite surface larger than $d_{50} 100 \mu \mathrm{m}$ (for the surface resistivity of the PVP-assisted silica@graphite, $d_{50}$ size 100, $300 \mu \mathrm{m}$, via the twostep process, see ESI $\uparrow$ ). Considering that silica species cannot grow individually on a large basal-plane with Triton X-100, the surface resistivity of the Triton X-assisted silica@graphite depends on the size of the graphite. SDS-assisted silica@graphite and lignin-assisted silica@graphite, which have rough morphology and some channels, have surface resistances of $10^{11}$ and $10^{8} \mathrm{ohm} \mathrm{sq}{ }^{-1}$, respectively. The combination of SDS/ PVP-assisted graphite, in which PVP has a more dominant position than SDS, has a surface resistivity of $10^{2} \mathrm{ohm} \mathrm{sq}^{-1}$. This value is similar to that of the PVP-assisted silica@graphite. The Triton-X/PVP-assisted silica@graphite attains a higher surface resistivity (in the range of $10^{12}$ to $10^{13} \mathrm{ohm} \mathrm{sq}{ }^{-1}$ ) through synergy than through Triton X-100. The PEG-assisted silica@graphite shows electrically insulating properties $\left(10^{11}\right.$ to $10^{12} \mathrm{ohm} \mathrm{sq}^{-1}$ ) in a broad range of graphite sizes: $d_{50} 3$ to 300 $\mu \mathrm{m}, d_{90} 6$ to $550 \mu \mathrm{m}$.

Fig. 9b shows the most appropriate range of PEG molecular weights is $M_{\mathrm{n}} 2050-4600 \mathrm{~g} \mathrm{~mol}^{-1}$ (for FE-SEM images of the surfaces of PEG-assisted silica@graphite depending on the size of the graphite and $M_{\mathrm{n}}$ of PEG, see ESI $\dagger$ ). PEG with a molecular weight of $M_{\mathrm{n}} 400 \mathrm{~g} \mathrm{~mol}^{-1}$ had very weak VDW interactions, while PEG with a molecular weight of $M_{\mathrm{n}} 10000 \mathrm{~g} \mathrm{~mol}^{-1}$ (lacking hydroxyl groups) cannot show satisfactory performance (it had a surface resistivity of $10^{2}$ to $10^{3} \mathrm{ohm} \mathrm{sq}^{-1}$ except in the case of $M_{\mathrm{n}} 400 \mathrm{~g} \mathrm{~mol}^{-1}$ and a $d_{50}$ size of $12 \mu \mathrm{m}$ ). As a result, Triton X-100 was only selected as a suitable silica-coating amphiphile for the $12 \mu \mathrm{m}$ graphite, while PEG $\left(M_{\mathrm{n}} 2050-\right.$ $4600 \mathrm{~g} \mathrm{~mol}^{-1}$ ) can be used as a coating over the broadest spectrum of graphite.
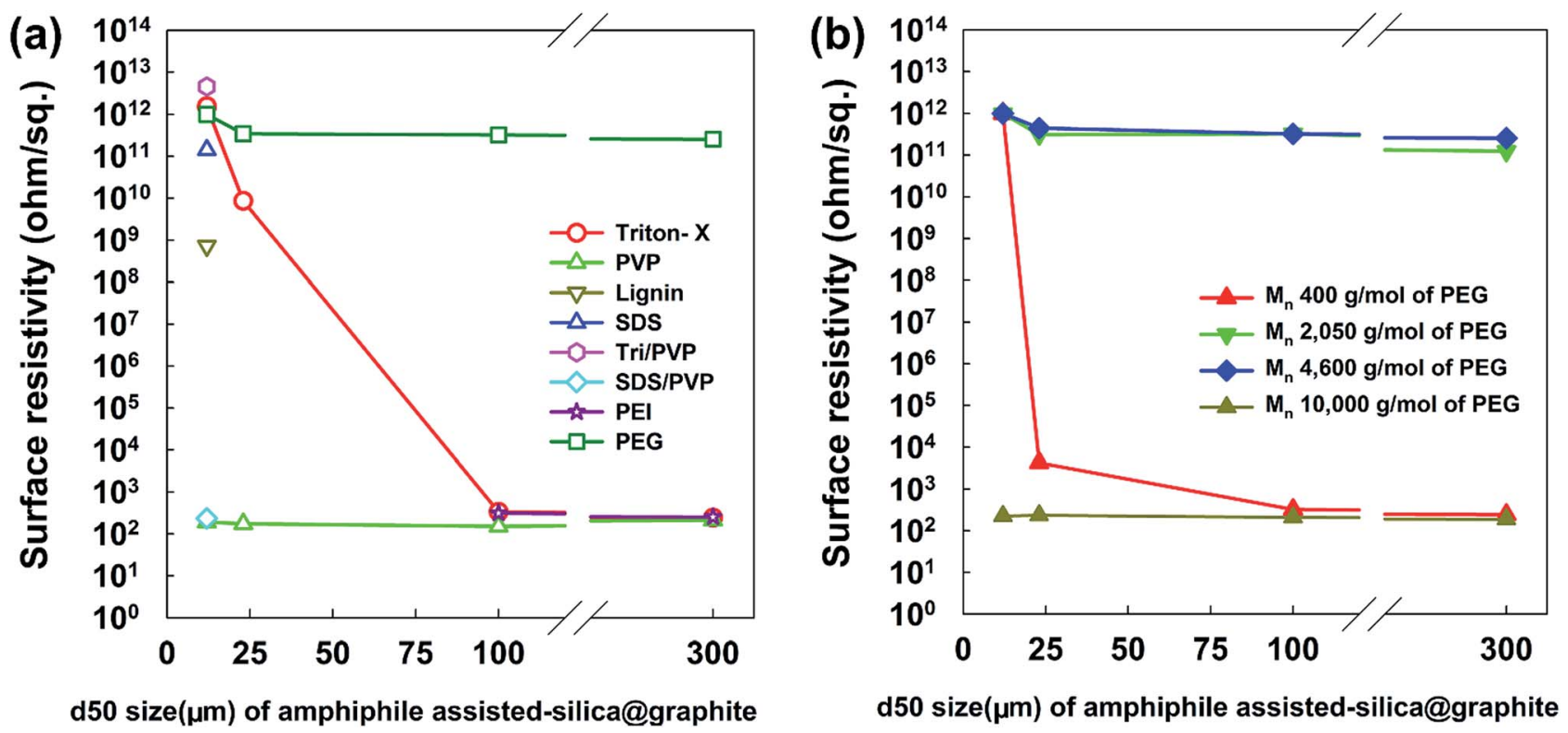

Fig. 9 Surface resistivity of amphiphile-assisted silica@graphite depending on the amphiphile and size $\left(d_{50}\right)$ of graphite. 
3.2.2. Effect of sol-gel reaction time on the surface resistivity of amphiphile-assisted silica@graphite. As shown in Fig. 10, this work helps assess (1) the optimal sol-gel reaction time for imparting electrically insulating properties, (2) the effect of the growth model on insulating efficiency (the surface resistivity per the sol-gel reaction time), and (3) whether the edge growth of silica species in this system intervenes in overall growth at a meaningful level. To endow the $12 \mu \mathrm{m}$ Triton-X-assisted silica@graphite, $12 \mu \mathrm{m}$ PEG-assisted silica@graphite, and $100 \mu \mathrm{m}$ PEG-assisted silica@graphite with electrically insulting properties $\left(10^{11}\right.$ to $\left.10^{13} \mathrm{ohm} \mathrm{sq}{ }^{-1}\right)$, the required sol-gel reaction times are 12,1 , and $3 \mathrm{~h}$, respectively. The growth of silica species for the PEG-assisted silica@graphite, which follows the Frank-van der Merwe growth model/Volmer-Weber growth model hybrid, is excellent for coating the basal plane with an electrically insulating layer. In view of the underlying mechanisms, the sol-gel reaction time for coatings can be reduced. The growth of the silica species is layer growth without electrically conductive channels; hence, it is advantageous for coating a uniform silica layer. The diversification of growth routes and growing points facilitates the growth of silica species on the graphite. In contrast, the surface resistivity of the Triton-X-assisted silica@graphite jumps after $9 \mathrm{~h}$ of reaction, because of the time necessary to fill the many channels on the basal plane with a silica layer (according to the Volmer-Weber growth model). A comparison of the surface resistivity of the $12 \mu \mathrm{m}$ PEG-assisted silica@graphite to that of the $100 \mu \mathrm{m}$ PEG-assisted silica@graphite showed that the surface energy depended on graphite size and the support of the formed nuclei on the edge led to a $2 \mathrm{~h}$ reduction (from $3 \mathrm{~h}$ to $1 \mathrm{~h}$ ) in the sol-gel reaction time.

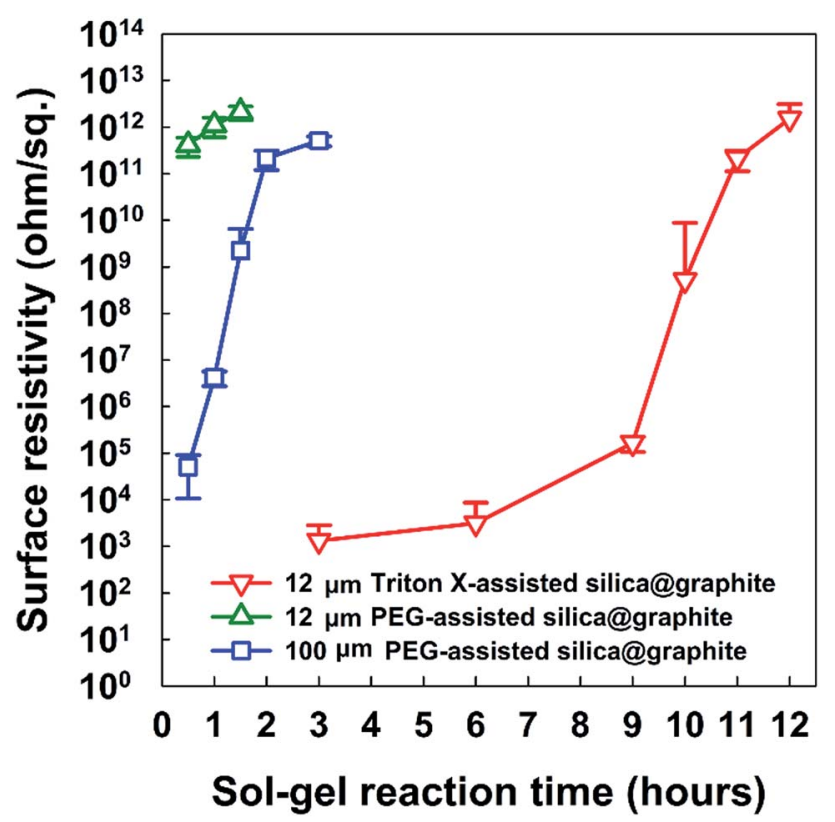

Fig. 10 Surface resistivity of Triton X-assisted silica@graphite $(12 \mu \mathrm{m})$, PEG-assisted silica@graphite (12 $\mu \mathrm{m})$, and PEG-assisted graphite (100 $\mu \mathrm{m})$ vs. sol-gel reaction time.
3.2.3. Surface resistivity and quantitative analysis of amphiphile-assisted silica@graphite. Fig. 11 (Fig. 11a and b: Triton-X-assisted silica@graphite; Fig. 11c and d: PEG-assisted silica@graphite) describes the correlation between particle diameters and layer thicknesses, coating morphology (TEM micrograph), the amount of silica (TGA), and the surface resistivity for each growth stage. In the case of Triton-X-assisted silica-growth (Fig. 11a and b), the diameter of the nuclei grows rapidly to approximately $40 \mathrm{~nm}$ in the b-3 stage, but many channels between the nuclei cannot electrically insulate the graphite. The channels are reduced when the nuclei and islands overlap in the b-4 stage; the Triton-X-assisted graphite possesses an electrically insulating property of $10^{10}$ to $10^{12} \mathrm{ohm}$ $\mathrm{sq}^{-1}$. To form a similar diameter of nuclei and similar layer thickness, the $12 \mu \mathrm{m}$ graphite requires almost twice the amount of silica as the $23 \mu \mathrm{m}$ graphite. This difference results from the surface area and particle size. In the PEG-assisted system (Fig. 11c and d), the "silica sol-PEG" network resulting from the use of PEG as a bridgemer enables the growth of silica species toward the $Z$-axis. Their random binding on the graphite appears for a certain amount of time to follow a 'layer by layer model', such as a Frank-van der Merwe mode. Therefore, from 0 to $3 \mathrm{~h}$ the thickness of the silica species on the graphite is proportional to the time. Although the growing layer has no channels, it does not become electrically insulating until its thickness exceeds 40-45 $\mathrm{nm}$.

Overall, to endow graphite with electrically insulating properties via a silica coating, the coated layer must have a surface devoid of channels and a thickness greater than $40-45 \mu \mathrm{m}$.

\subsection{Thermal conductivity and surface resistivity of silica@graphite/TPEE composites}

Fig. 12 shows the thermal conductivity and surface resistivity of both raw graphite/TPEE composites and amphiphile-assisted silica@graphite/TPEE composites with $50 \mathrm{wt} \%$ filler content. The silica layer on the graphite acts as a thermal and electrical barrier (the thermal conductivity of silica is $1.5 \mathrm{~W} \mathrm{mK}^{-1}$ (ref. 29)).

The silica layer reduces the thermal conductivity of the composites to $70-75 \%$ of their original values $(12 \mu \mathrm{m}$ graphite: $70 \%, 23 \mu \mathrm{m}$ graphite: 75\%). The difference in reduction results from the overall number of silica species and the number of total barriers per volume of the composite. In the case of the $12 \mu \mathrm{m}$ amphiphile-assisted silica@graphite/TPEE $50 \mathrm{wt} \%$ composite, electrically insulating properties were successfully attained $\left(10^{11}\right.$ to $10^{12} \mathrm{ohm} \mathrm{sq}^{-1}$ ); however, the $23 \mu \mathrm{m}$ amphiphile-assisted silica@graphite/TPEE composite lost its insulating properties $\left(10^{7}\right.$ to $\left.10^{8} \mathrm{ohm} \mathrm{sq}{ }^{-1}\right)$. This means that the silica layer on the graphite is ground against the molten polymer resin, which has a high melt viscosity during the compounding process. The 12 $\mu \mathrm{m}$ amphiphile-assisted silica@graphite has similar thickness to the $23 \mu \mathrm{m}$ amphiphile-assisted silica@graphite, and the same silica species; however, there is an approximately twofold difference in the number of silica species. In the case of the $12 \mu \mathrm{m}$ amphiphile-assisted silica@graphite/TPEE composite, the abundance of crushed splinters interrupts the electrical flow 
(a)

\begin{tabular}{|c|c|c|c|c|}
\hline \multirow{2}{*}{$\begin{array}{l}\text { The feature of the coating } \\
\text { morphology }\end{array}$} & \multirow{2}{*}{$\begin{array}{l}\text { The diameter of the } \\
\text { particle or the } \\
\text { thickness of the layer } \\
\text { by TEM }(\mathrm{nm})\end{array}$} & \multicolumn{2}{|c|}{$\begin{array}{l}\text { The amount of silica by TGA } \\
\text { (mass } \% \text { to total) }\end{array}$} & \multirow{2}{*}{$\begin{array}{l}\text { Surface resistivity } \\
\text { (Ohm/sq.) }\end{array}$} \\
\hline & & Graphite of $12 \mu \mathrm{m}$ & Graphite of $23 \mu \mathrm{m}$ & \\
\hline (b-1) Formation of nuclei & $5-20$ & $5-10$ & $2-5$ & $10^{2}-10^{3}$ \\
\hline $\begin{array}{l}\text { (b-2) Grown nuclei } \\
\text { (b-3) Well-grown nuclei }\end{array}$ & $20-45$ & $15-18$ & $7-9$ & $10^{5}-10^{7}$ \\
\hline $\begin{array}{l}\text { (b-4) Condensed islands } \\
\text { (b-5) A dense layer }\end{array}$ & $45-60$ & $21-25$ & $10-17$ & $10^{10}-10^{12}$ \\
\hline
\end{tabular}

(b)
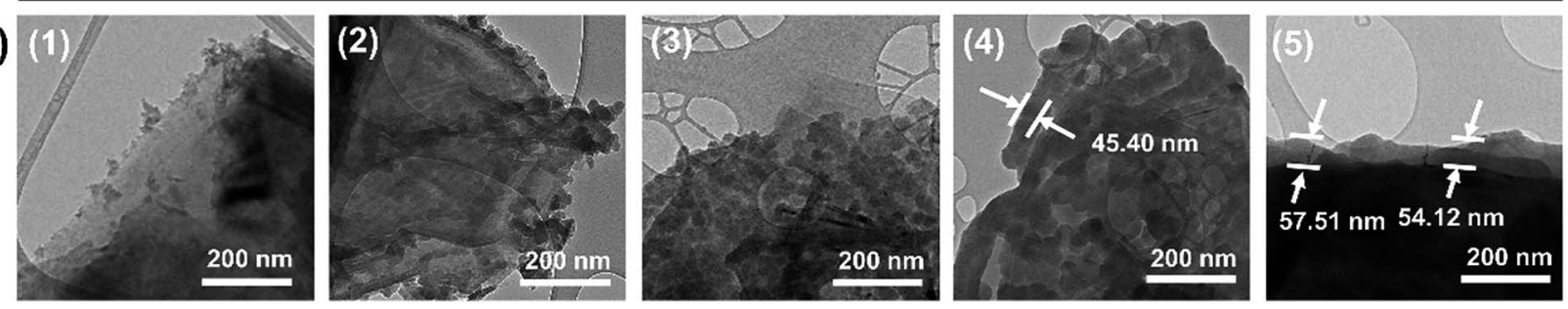

(c)

\begin{tabular}{|c|c|c|c|}
\hline \multirow{2}{*}{$\begin{array}{l}\text { The feature of the coating } \\
\text { morphology }\end{array}$} & \multirow{2}{*}{$\begin{array}{l}\text { The diameter of the } \\
\text { particle or the } \\
\text { thickness of the layer } \\
\text { by TEM }(\mathrm{nm})\end{array}$} & $\begin{array}{l}\text { The amount of silica by TGA } \\
\text { (mass } \% \text { to total) }\end{array}$ & \multirow{2}{*}{$\begin{array}{l}\text { Surface resistivity } \\
\text { (Ohm/sq.) }\end{array}$} \\
\hline & & $100 \mu \mathrm{m}$ of graphite & \\
\hline (d-1) A thin layer & $5-15$ & $2-3$ & $10^{3}-10^{4}$ \\
\hline \multirow{2}{*}{$(d-2)(d-3)$ A grown layer } & $20-30$ & $3-4$ & $10^{5}-10^{7}$ \\
\hline & $30-45$ & 4-6 & $10^{7}-10^{9}$ \\
\hline (d-4) A dense and smooth layer & $45-65$ & $6-8$ & $10^{10}-10^{12}$ \\
\hline
\end{tabular}

(d)
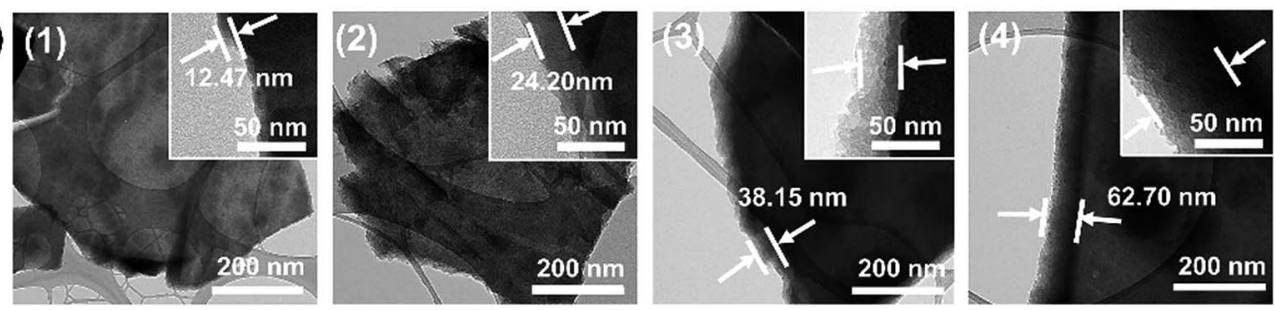

Fig. 11 (a) Surface resistivity, coating morphology features, particle diameter or thickness, amount of silica for the Triton-X-100-assisted silica@graphite (12 and $23 \mu \mathrm{m}$ ) and (b) TEM images. (c) Surface resistivity, coating morphology features, particle diameter or thickness, amount of silica for the PEG-assisted silica@graphite $(100 \mu \mathrm{m})$ and (d) TEM images (for original TGA graphs, see ESI $\dagger$ ).

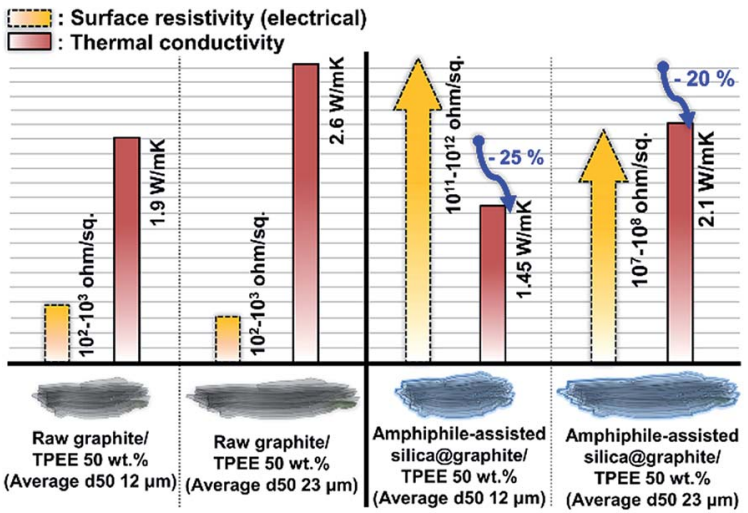

Fig. 12 Thermal conductivity and surface resistivity of raw graphite ( $d_{50}$ size: 12 and $\left.23 \mu \mathrm{m}\right)$ and amphiphile-assisted silica@graphite $\left(d_{50}\right.$ size: 12 and $\left.23 \mu \mathrm{m}\right) /$ TPEE composites with 50 wt\% filler content. pathways between the damaged amphiphile-assisted silica@graphite in the polymer matrix.

\section{Conclusion}

Amphiphile-assisted silica@graphite with uniform full coverage was synthesized using an Oswald ripening agent, such as Triton $\mathrm{X}-100$, and a bridgemer, such as PEG, via a modified one-step process. The mechanism of amphiphile-assisted silica formation on graphite was elucidated, and three suitable strategies were suggested, depending on the graphite size. The Oswald ripening agent provides nuclei surrounded by embryo-forming functional groups; it facilitates the absorption of embryos by the nuclei. This behavior assists the formation of islands and layers from the nuclei through Oswald ripening. For example, with Triton-X-100, the silica species grow from the edge toward the basal plane, and the assistance helps fill the many channels with silica species. The bridgemer couples the sol masses and 
binds the networks onto the other material substrates. For example, PEG can facilitate the growth of silica species toward the $Z$-axis, without the existence of adjacent nuclei and islands on the basal plane and edge. Consequently, the amphiphileassisted silica@graphite can become electrically insulating $\left(10^{11}\right.$ to $\left.10^{12} \mathrm{ohm} \mathrm{sq}^{-1}\right)$. The TPEE composite containing $50 \mathrm{wt} \%$ filler shows electrically insulating properties $\left(10^{11}\right.$ to $10^{12} \mathrm{ohm}$ $\mathrm{sq}^{-1}$ ) and $70-75 \%$ of the thermal conductivity of a composite made of equal parts raw graphite/TPEE. Finally, the amphiphile-assisted silica@graphite can be applied in various fields. Moreover, the coating strategies have potential to can coat not only graphite, but also other cabonaceous materials, such as graphene and carbon nanotubes.

\section{Acknowledgements}

This work was supported by a grant [1415140523/10052976, 2015] from the Korea Ministry of Trade, Industry and Energy (MOTIE), Republic of Korea.

\section{Notes and references}

1 B. Lee, J. Liu, B. Sun, C. Shen and G. Dai, eXPRESS Polym. Lett., 2008, 2, 57-63.

2 S. Ganguli, A. K. Roy and D. P. Anderson, Carbon, 2008, 46, 806-817.

3 K. Kalaitzidou, H. Fukushima and L. T. Drzal, Compos. Sci. Technol., 2007, 67, 2045-2051.

4 P. K. Schelling, L. Shi and K. E. Goodson, Mater. Today, 2005, 8, 30-35.

5 D. G. Cahill, W. K. Ford, K. E. Goodson, G. D. Mahan, A. Majumdar, H. J. Maris, et al., J. Appl. Phys., 2003, 93, 793-818.

6 W. Cui, F. Du, J. Zhao, W. Zhang, Y. Yang, X. Xie, et al., Carbon, 2011, 49, 495-500.

7 A. Guimont, E. Beyou, P. Alcouffe, P. Cassagnau, A. Serghei, G. Martin, et al., Polymer, 2014, 55, 22-28.

8 S. H. Song, K. H. Park, B. H. Kim, Y. W. Choi, G. H. Jun, D. J. Lee, et al., Adv. Mater., 2013, 25, 732-737.

9 L. Cao, W. Zhang, X. Zhang, L. Yuan, G. Liang and A. Gu, Ind. Eng. Chem. Res., 2014, 53, 2661-2672.

10 F. He, K. Lam, J. Fan and L. H. Chan, Carbon, 2014, 80, 496503.

11 C. Min, D. Yu, J. Cao, G. Wang and L. Feng, Carbon, 2013, 55, 116-125.

12 Y. Kim, M. Kim, J. K. Choi and S. E. Shim, Polymer, 2015, 39, 136-143.
13 T. Matsuda, D. Minami, F. Khoerunnisa, M. Sunaga, M. Nakamura, S. Utsumi, et al., Langmuir, 2015, 31, 31943202.

14 M. Kim, Y. Kim, S. H. Baeck and S. E. Shim, Carbon letters, 2015, 16, 34.

15 J. Hong, J. Lee, C. K. Hong and S. E. Shim, J. Therm. Anal. Calorim., 2010, 101, 297-302.

16 K. Kim, Y. Kim, J. Nam, S.-H. Baeck, D. W. Park and S. E. Shim, Polymer, 2016, 40, 117-123.

17 J. Nam, Y. Kim, K. Kim, S.-H. Baeck, D. W. Park and S. E. Shim, Polymer, 2016, 40, 109-116.

18 S. Choi, Y. Kim, J. H. Yun, I. Kim and S. E. Shim, Mater. Lett., 2013, 90, 87-89.

19 S. Choi, K. Kim, J. Nam and S. E. Shim, Carbon, 2013, 60, 254-265.

20 S. Choi, J. Yang, Y. Kim, J. Nam, K. Kim and S. E. Shim, Compos. Sci. Technol., 2014, 103, 8-15.

21 M.-C. Hsiao, C.-C. M. Ma, J.-C. Chiang, K.-K. Ho, T.-Y. Chou, X. Xie, et al., Nanoscale, 2013, 5, 5863-5871.

22 D. Tang, J. Su, Q. Yang, M. Kong, Z. Zhao, Y. Huang, et al., RSC Adv., 2015, 5, 55170-55178.

23 Y. Noma, Y. Saga and N. Une, Carbon, 2014, 78, 204-211.

24 H. Yang, N. Coombs, I. Sokolov and G. A. Ozin, J. Mater. Chem., 1997, 7, 1285-1290.

25 C. I. Zoldesi, P. Steegstra and A. Imhof, J. Colloid Interface Sci., 2007, 308, 121-129.

26 Y. Qu, Y. Tian, B. Zou, J. Zhang, Y. Zheng, L. Wang, et al., Bioresour. Technol., 2010, 101, 8402-8405.

27 W. Guo, Y. W. Sun, G. S. Luo and Y. J. Wang, Colloids Surf., A, 2005, 252, 71-77.

28 L. L. Hench and J. K. West, Chem. Rev., 1990, 90, 33-72.

29 G.-W. Lee, M. Park, J. Kim, J. I. Lee and H. G. Yoon, Composites, Part A, 2006, 37, 727-734.

30 Y.-S. Yoon, M.-H. Oh, A.-Y. Kim and N. Kim, J. Chem. Chem. Eng., 2012, 6, 515-519.

31 M. A. Herman, W. Richter and H. Sitter, Epitaxy: Physical Principles and Technical Implementation, Springer-Verlag, Berlin, 2004, pp. 6-12.

32 J. T. Davies, A quantitative kinetic theory of emulsion type, I. Physical chemistry of the emulsifying agent, Proceedings of 2nd the International Congress of Surface Activity, Academic Press, New York, 1957, pp. 426-38.

33 B. Delmon, P. A. Jacobs, R. Maggi, J. A. Martens, P. Grange and G. Poncelet. Preparation of Catalysts VII., Louvain-laNeuve, Belgium, 1998, pp. 618-623.

34 Y. Pan, Y. Guo, X. Zhao and Z. Wang, Chem. Res. Chin. Univ., 2012, 28, 737-742. 